\title{
Extending the Limits of Rotationally Resolved Absorption Spectroscopy: Pyrene
}

\author{
Brian E. Brumfield, ${ }^{\dagger}$ Jacob T. Stewart, and Benjamin J. McCall* \\ Department of Chemistry, University of Illinois, Urbana, Illinois 61801, United States
}

\section{Supporting Information}

ABSTRACT: We report the rotationally resolved gas phase spectrum of pyrene $\left(\mathrm{C}_{16} \mathrm{H}_{10}\right)$, which is now the largest molecule to be observed with rotational resolution using absorption spectroscopy. This represents a significant advance in the application of absorption spectroscopy to large carbon-containing molecules of fundamental chemical and astronomical importance. Such spectra will facilitate the search for large and highly symmetric molecules in interstellar space, where they may be abundant but cannot be detected without the support of highresolution laboratory spectra. Detailed assignment and analysis of our spectrum indicates that pyrene is the most rigid rotor yet observed, and that a supersonic expansion cools both the rotational and vibrational temperatures of pyrene vapor produced in an oven source.

SECTION: Spectroscopy, Photochemistry, and Excited States

$\mathrm{T}$ he field of astrochemistry seeks insight into the rich, diverse, and unique chemistry occurring in remote regions of the universe where environmental conditions differ radically from those encountered in a terrestrial laboratory. Understanding the possible chemical pathways that can lead from simple molecular precursors, such as acetylene, to large potentially prebiotic molecules in space is an important challenge in the field. To piece together this puzzle, it is necessary to identify molecules in space through observation of their unique absorption or emission features. The identity, temperature, and density of the surrounding chemical environment can be obtained from astronomical spectra, but only after molecules are identified with the aid of laboratory spectra. In particular, high-resolution gas phase molecular spectroscopy is an invaluable tool that can enable unequivocal detection of molecules in space. While many small (e.g., $\mathrm{H}_{2}, \mathrm{H}_{3}{ }^{+}$, and $\mathrm{CO})^{1-3}$ and intermediate size molecules (e.g., $\mathrm{C}_{2} \mathrm{H}_{2}, \mathrm{CH}_{4}, \mathrm{C}_{5}$, and $\left.\mathrm{C}_{6} \mathrm{H}_{6}\right)^{4-7}$ have been detected in space and identified with the aid of laboratory spectra, there is a lack of high-resolution spectra of large potentially prebiotic molecules; this presents a significant challenge to identifying such molecules in space, and the field of high-resolution molecular spectroscopy must rise to this challenge. High-resolution studies of large molecules are also important from a fundamental chemical standpoint, as analysis of such spectra can provide insight into the structure and dynamics of large molecules.

A majority of astronomical observations of molecules are made using microwave or millimeter-wave spectroscopy of pure rotational transitions of molecules with permanent electric dipole moments. However, there are many large carbon containing molecules of potential prebiotic significance, such as linear carbon chains, symmetric fullerenes, and polycyclic aromatic hydrocarbons (PAHs) that lack a permanent dipole moment and can only be observed by vibrational or electronic spectroscopy. Linear carbon chains have already been detected astronomically in space, and PAHs of extraterrestrial origin have been identified in meteorites. ${ }^{8}$ However, acquiring highresolution gas-phase spectra of large molecules using direct absorption spectroscopy is challenging because these molecules tend to have low vapor pressures at room temperature, and usually require direct heating or laser ablation techniques to put a sufficient number density of the sample into the gas phase. $^{9-14}$ The rotational and vibrational temperatures of these hot molecules can be cooled using the well-developed supersonic expansion technique. Large symmetric PAHs, such as pyrene $\left(\mathrm{C}_{16} \mathrm{H}_{10}\right)$ or coronene $\left(\mathrm{C}_{24} \mathrm{H}_{12}\right)$, present ideal challenges for pushing the limit of these experimental techniques and enabling new astronomical detections. Although laser-induced fluorescence (LIF) studies have shown the effectiveness of vibrational cooling of large organic molecules using pulsed sources, ${ }^{9,10,24}$ there is little information about the degree of vibrational cooling achievable using a heated cw supersonic expansion source for absorption spectroscopy studies.

In this Letter, we present the rotationally resolved absorption spectrum of a $\mathrm{C}-\mathrm{H}$ bending mode of pyrene, which is the largest molecule to be observed with rotational resolution by absorption spectroscopy. The necessity of good vibrational cooling in the supersonic expansion is also discussed, and an

Received: June 13, 2012

Accepted: July 11, 2012 
estimate for the vibrational temperature is presented. This work represents a significant step in high-resolution absorption spectroscopy of large carbon-containing and potentially prebiotic molecules.

Pyrene is a planar asymmetric rotor of $D_{2 h}$ point group symmetry, and the $\nu_{68}$ vibrational mode studied here has $B_{3 u}$ symmetry and involves $\mathrm{C}-\mathrm{H}$ bending in concert with an inplane displacement of the two central carbon atoms in the fused-ring structure (see Figure 1). Low-resolution absorption

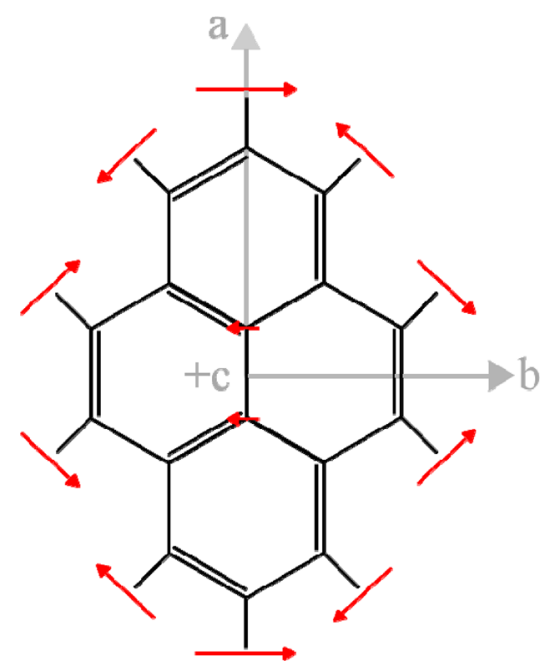

Figure 1. The structure and principal axes $(a, b, c)$ of pyrene with arrows illustrating the displacement of the nuclei associated with the observed $\nu_{68} \mathrm{C}-\mathrm{H}$ bending mode.

spectra of this $\mathrm{C}-\mathrm{H}$ bending mode have been obtained ${ }^{15,16}$ in rare gas matrices and in high-temperature gas phase samples. These data predict that the vibrational band center for the $\mathrm{C}-$ $\mathrm{H}$ bending mode will be near $1184 \mathrm{~cm}^{-1}(8.44 \mu \mathrm{m})$, and this is close to a widely observed astronomical feature at $8.6 \mu \mathrm{m}$, part of the so-called unidentified infrared bands. ${ }^{17}$ While the matrix and hot gas phase spectra of pyrene are helpful for guiding a high-resolution spectroscopic search, they are inadequate for definitive comparisons to high-resolution infrared astronomical spectra. The best mid-IR spectrographs are capable of 0.01 $\mathrm{cm}^{-1}$ resolution in comparison to the $1 \mathrm{~cm}^{-1}$ resolution of the matrix and hot gas phase measurements. Additionally, the shift in the vibrational band center due to interactions with the raregas matrix can be on the order of several wavenumbers, and in the astronomical environment where spectral congestion can be high, such an uncertainty in the vibrational band center is unacceptable. The resolution of the current study is $<0.001$ $\mathrm{cm}^{-1}$, providing more resolution than is necessary to meet the requirements for astronomical searches.

The $\nu_{68} \mathrm{C}-\mathrm{H}$ bending mode was probed using a quantum cascade laser-based continuous-wave cavity ringdown spectrometer coupled to a heated supersonic expansion source that was operated continuously while collecting experimental spectra. Details regarding the spectrometer have been presented in two prior publications, ${ }^{18,19}$ and details regarding the heated supersonic expansion source can be found in the Supporting Information. Briefly, argon gas was passed through a heated $(420 \mathrm{~K})$ oven containing pyrene, and the mixture was supersonically expanded through a slit nozzle. The expansion was overlapped with the ringdown cavity axis $6 \mathrm{~mm}$ downstream from the nozzle.

Through temperature and current tuning of the laser, it was possible to record the pyrene vibrational band from 1182.77 $1185.06 \mathrm{~cm}^{-1}$, as displayed in Figure 2. Our spectra were calibrated to an accuracy of $0.00049 \mathrm{~cm}^{-1}(15 \mathrm{MHz})$ using $\mathrm{SO}_{2}$ absorption line features and a wavemeter; further information on the calibration procedures is in the Supporting Information. The observed line width for transitions in the supersonic expansion was $0.0004 \mathrm{~cm}^{-1}(12 \mathrm{MHz})$, which is sufficient to resolve the detailed rotational structure of the vibrational band.

To extract quantitative information on the structure of pyrene, we assigned the line center frequency positions for 694 observed lines (representing 2222 rotation-vibration transitions). The line center assignments were then used in a leastsquares fitting optimization routine to a model asymmetric top Hamiltonian using PGOPHER. ${ }^{20}$ Initially, we constrained the values of the ground state rotational constants to those obtained from a fluorescence excitation study of an electronic band of pyrene. ${ }^{21}$ However, this failed to produce good agreement over the entire spectrum (see Supporting Information), so it was necessary to fit both ground and excited state rotational constants. The derived molecular constants are listed in Table 1, and the standard deviation of the residuals to the fit is only $0.00053 \mathrm{~cm}^{-1}(16 \mathrm{MHz})$. The

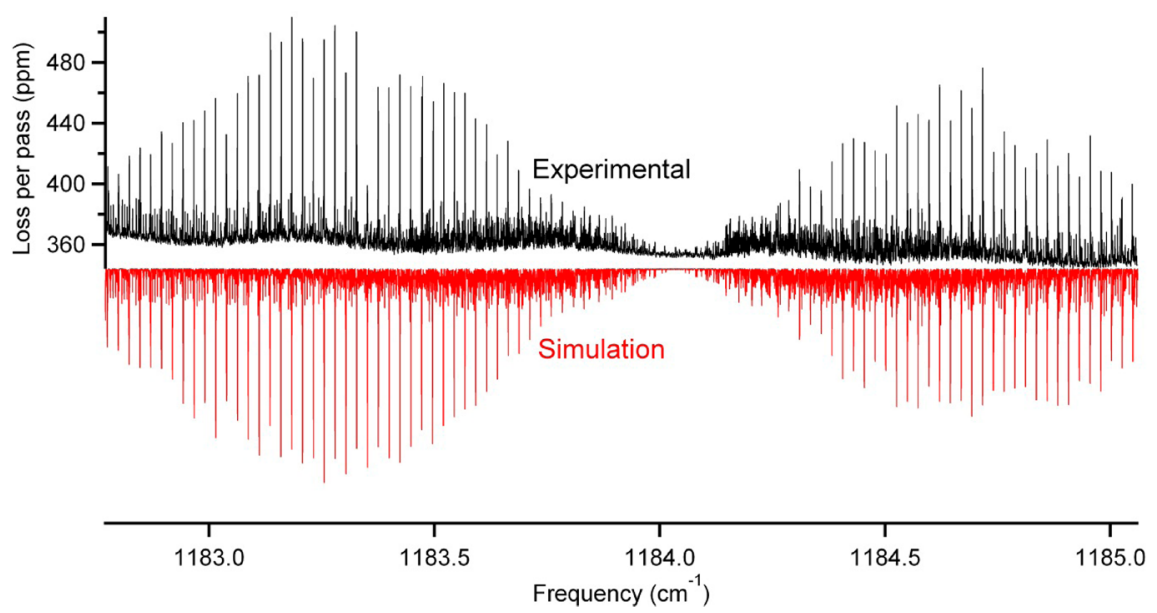

Figure 2. Cavity ringdown spectrum of the $\nu_{68}$ band of pyrene (top trace, in black) in units of fractional loss per-pass (ppm) versus frequency $\left(\mathrm{cm}^{-1}\right)$, compared with simulated spectrum (lower trace, in red). 
Table 1. Derived Molecular Constants (in $\mathrm{cm}^{-1}$, with Uncertainties in Last Digits in Parentheses)

\begin{tabular}{cccc} 
& ground state & excited state & percent change \\
\hline$\nu_{0}$ & & $1184.035595(20)$ & \\
$A$ & $0.03372547(66)$ & $0.03372116(67)$ & $-0.0128(28) \%$ \\
$B$ & $0.01855623(43)$ & $0.01855635(43)$ & $+0.0006(33) \%$ \\
$C$ & $0.01197350(33)$ & $0.01197172(34)$ & $-0.0149(40) \%$ \\
\hline
\end{tabular}

final simulation is in excellent agreement with the recorded spectrum, as seen for the entire band in Figure 2 and for a few select regions in Figure 3 (an expanded view of the entire spectrum is available in the Supporting Information).

One important result of this work is that the change in the rotational constants upon vibrational excitation is quite small: the relative changes for $A, B$, and $C$ are $-0.0128 \%, 0.0006 \%$, and $-0.0149 \%$, respectively. The change in $B$ is so small that it is not statistically significant, given the uncertainties in the fit. Molecules similar to pyrene, such as pyrazine and naphthalene, have been observed to have small changes in rotational constants when vibrationally excited, ${ }^{22}$ but pyrene is remarkable in that all three constants change by less than $0.02 \%$ in the excited vibrational state. The rotational constant of adamantane, which has a more compact structure than pyrene but the same number of atoms, also exhibits larger changes in its rotational constant upon vibrational excitation. ${ }^{23}$

Another exceptional aspect of this result is that no centrifugal distortion constants were required to fit the spectrum. In a trial fit, we added a $D_{\mathrm{K}}$ constant, but the standard deviation only decreased by $0.00004 \mathrm{~cm}^{-1}$, which we do not consider significant given the accuracy of our frequency calibration using $\mathrm{SO}_{2}$. The fact that the spectrum can be fit without any distortion constants, combined with the very small changes in rotational constants upon vibrational excitation, shows that pyrene comes close to behaving like an ideal rigid asymmetric rotor.

By comparing the relative intensities of the observed spectral lines with simulations at various temperatures, we were able to estimate the rotational temperature of the pyrene in our supersonic expansion to be $\approx 23 \mathrm{~K}$. (for more details, see Supporting Information). This effective rotational cooling from the source temperature of $\approx 420 \mathrm{~K}$ is consistent with the wellknown efficiency of rotational-to-translational energy transfer in expansions. $^{9}$

A driving motivation of the current work was to explore the efficiency of vibrational relaxation of such a large molecule in a supersonic expansion from a heated source. Vibrational cooling is critical because absorption from the ground vibrational state to an excited state is most relevant for enabling astronomical detections of molecules in the low temperature conditions of interstellar space. Adequate vibrational cooling is also experimentally important because of the strong temperature dependence of the vibrational partition function for large molecules. This occurs because large molecules have many vibrational modes and even at modest vibrational temperatures a significant portion of the molecular population can be partitioned into excited vibrational states. The concomitant depletion of population from the ground vibrational state reduces the absorption signal, potentially rendering observation of the spectrum technically infeasible even with sensitive absorption spectroscopy techniques. However, transfer of energy from vibrational degrees of freedom into rotation and translation is generally less efficient than rotation-translation transfer. For pyrene, with 72 vibrational normal modes, if there were no vibrational relaxation from the initial source temperature of $420 \mathrm{~K}$, only 1 in 3000 pyrene molecules would be in the ground vibrational state. At a vibrational temperature of 50 $\mathrm{K}$, in contrast, most of the pyrene molecules in the expansion are in the ground state.

To evaluate the vibrational cooling of pyrene, we used the quantitative absorption strength information from the cavity ringdown spectra. By calculating the total number density of pyrene in the cavity, and comparing the expected absorption intensity with that observed (see Supporting Information for details), we were able to infer the vibrational partition function $\left(Q_{v i b}\right)$ to be $1.41 \pm 0.17$. This corresponds to a $3 \sigma$ upper bound on the vibrational temperature of $111 \mathrm{~K}$; we consider the rotational temperature of $23 \mathrm{~K}$ to be the lower bound of the vibrational temperature. This is consistent with vibrational temperature estimates from LIF studies of large organic molecules seeded in heated supersonic expansion sources. $9,10,24$
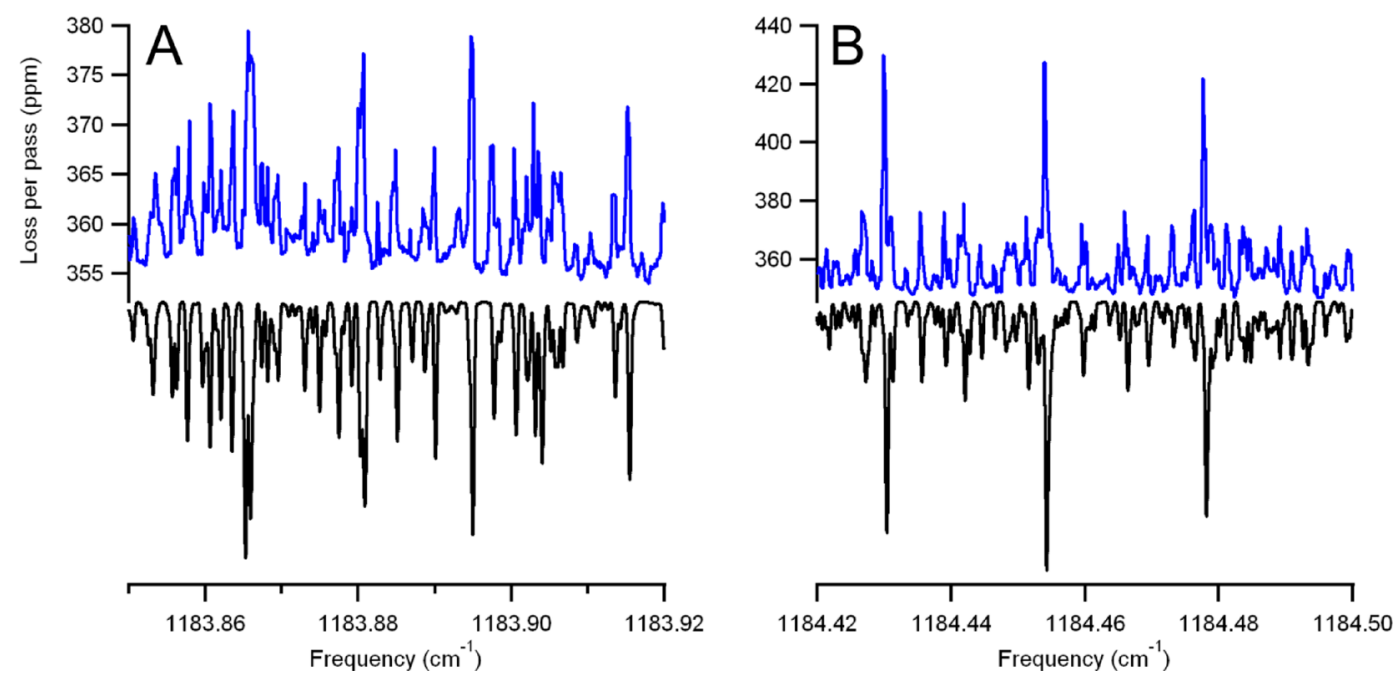

Figure 3. Expanded views of the observed (top traces, blue) and simulated (lower traces, black) spectrum near the band center (panel A), and in the R-branch (panel B). 
In summary, pyrene is now the largest molecule to be studied with rotational resolution by absorption spectroscopy, and this work illustrates the feasibility of high-resolution large molecule spectroscopy using a simple heated oven supersonic expansion source. Analysis of the spectrum led to extraction of rotational constants for the ground and vibrationally excited states, revealing the extraordinarily rigid molecular structure of pyrene, in agreement with previous knowledge regarding the relative stiffness of PAHs. Larger PAHs, like coronene $\left(\mathrm{C}_{24} \mathrm{H}_{16}\right)$, are ideal targets to continue to test the limits of high-resolution absorption spectroscopy of large molecules, and to study the effectiveness of vibrational cooling in a slit nozzle supersonic expansion. Knowledge gained from these studies will offer insight into the feasibility of rotationally resolved infrared absorption spectroscopy of large potentially prebiotic molecules and fullerenes such as $\mathrm{C}_{60}$ and $\mathrm{C}_{70}$. The resulting rotationally resolved spectra of PAHs and fullerenes will enable the first high-resolution astronomical searches for these large molecules in the interstellar medium.

\section{ASSOCIATED CONTENT}

\section{(5) Supporting Information}

Detailed comparison between experimental and simulated spectra, determination of rotational temperature, estimate of vibrational temperature, heated oven source details, frequency calibration, and a spectral line list are provided in the Supporting Information. This material is available free of charge via the Internet at http://pubs.acs.org.

\section{AUTHOR INFORMATION}

\section{Corresponding Author}

*E-mail: bjmccall@illinois.edu.

\section{Present Address}

${ }^{\dagger}$ Department of Electrical Engineering, Princeton University, Princeton, NJ 08544, United States.

\section{Author Contributions}

The manuscript was written through contributions of all authors.

\section{Notes}

The authors declare no competing financial interest.

\section{ACKNOWLEDGMENTS}

The authors wish to thank Matt Escarra and Professor Claire Gmachl from the Electrical Engineering Department at Princeton University for providing the quantum cascade laser used in this work. The development of our cavity ringdown spectrometer has been supported by the NASA Laboratory Astrophysics program and a David and Lucile Packard Fellowship. J.T.S. has been supported by a Robert C. and Carolyn J. Springborn Fellowship from the University of Illinois.

\section{REFERENCES}

(1) Carruthers, G. R. Rocket Observation of Interstellar Molecular Hydrogen. Astrophys. J. Lett. 1970, 161, L81-L85.

(2) Geballe, T. R.; Oka, T. Detection of $\mathrm{H}_{3}{ }^{+}$in Interstellar Space. Nature 1996, 384, 334-335.

(3) Wilson, R. W.; Jefferts, K. B.; Penzias, A. A. Carbon Monoxide in the Orion Nebula. Astrophys. J. 1970, 161, L43-L44.

(4) Lacy, J. H.; Evans, N. J., II.; Achtermann, J. M.; Bruce, D. E.; Arens, J. F.; Carr, J. S. Discovery of Interstellar Acetylene. Astrophys. J. 1989, 342, L43-L46.
(5) Lacy, J. H.; Carr, J. S.; Evans, N. J., II.; Baas, F.; Achtermann, J. M.; Arens, J. F. Discovery of Interstellar Methane - Observations of Gaseous and Solid $\mathrm{CH}_{4}$ Absorption Toward Young Stars in Molecular Clouds. Astrophys. J. 1991, 376, 556-560.

(6) Bernath, P. F.; Hinkle, K. H.; Keady, J. J. Detection of $\mathrm{C}_{5}$ in the Circumstellar Shell of IRC+10216. Science 1989, 244, 562-564.

(7) Cernicharo, J.; Heras, A. M.; Tielens, A. G. G. M.; Pardo, J. R.; Herpin, F.; Gualin, M.; Waters, L. B. F. M. Infrared Space Observatory's Discovery of $\mathrm{C}_{4} \mathrm{H}_{2}, \mathrm{C}_{6} \mathrm{H}_{2}$, and Benzene in CRL 618. Astrophys. J. Lett. 2001, 546, L123-L126.

(8) Botta, O.; Bada, J. L. Extraterrestrial Organic Compounds in Meteorites. Surv. Geophys. 2002, 23, 411-467.

(9) Amirav, A.; Even, U.; Jortner, J. Cooling of Large and Heavy Molecules in Seeded Supersonic Beams. Chem. Phys. 1980, 51, 31-42.

(10) Amirav, A.; Even, U.; Jortner, J. Absorption Spectroscopy of Ultracold Large Molecules in Planar Supersonic Expansions. Chem. Phys. Lett. 1981, 83, 1-4.

(11) Cable, J. R.; Tubergen, M. J.; Levy, D. H. Laser Desorption Molecular Beam Spectroscopy: The Electronic Spectra of Tryptophan Peptides in the Gas Phase. J. Am. Chem. Soc. 1987, 109, 6198-6199.

(12) Elam, J. W.; Levy, D. H. Laser Ablation of Trp-Gly. J. Phys. Chem. B 1998, 102, 8113-8120.

(13) Snoek, L. C.; van Mourik, T.; Simons, J. P. Neurotransmitters in the Gas Phase: A Computational and Spectroscopic Study of Noradrenaline. Mol. Phys. 2003, 101, 1239-1248.

(14) van Dantzig, N. A.; Piotrowiak, P.; Levy, D. H. van der Waals Complexes of the Bichromophore Spirobifluorene. Chem. Phys. Lett. 1994, 223, 127-132.

(15) Vala, M.; Szczepanski, J.; Pauzat, F.; Parisel, O.; Talbi, D.; Ellinger, Y. Electronic and Vibrational Spectra of Matrix-Isolated Pyrene Radical Cations: Theoretical and Experimental Aspects. J. Phys. Chem. 1994, 98, 9187-9196.

(16) Joblin, C.; Boissel, P.; Leger, A.; D’Hendecourt, L.; Defourneau, D. Infrared Spectroscopy of Gas-Phase PAH Molecules. II. Role of the Temperature. Astron. Astrophys. 1995, 299, 835-846.

(17) Allamandola, L. J.; Tielens, A. G. G. M.; Barker, J. R. Interstellar Polycyclic Aromatic Hydrocarbons: The Infrared Emission Bands, the Excitation/Emission Mechanism, and the Astrophysical Implications. Astrophys. J. Suppl. 1989, 71, 733-775.

(18) Brumfield, B. E.; Stewart, J. T.; Widicus Weaver, S. L.; Escarra, M. D.; Howard, S. S.; Gmachl, C. F.; McCall, B. J. A Quantum Cascade Laser CW Cavity Ringdown Spectrometer Coupled to a Supersonic Expansion Source. Rev. Sci. Instrum. 2010, 81, 063102.

(19) Brumfield, B. E.; Stewart, J. T.; McCall, B. J. High-Resolution Spectroscopy of the $\nu_{8}$ Band of Methylene Bromide Using a Quantum Cascade Laser. J. Mol. Spectrosc. 2011, 266, 57-62.

(20) Western, C. M. PGOPHER, a Program for Simulating Rotational Structure, http://pgopher.chm.bris.ac.uk, 2010.

(21) Baba, M.; Saitoh, M.; Kowaka, Y.; Taguma, K.; Yoshida, K.; Semba, Y.; Kasahara, S.; Yamanaka, T.; Ohshima, Y.; Hsu, Y.-C.; Lin, S. H. Vibrational and Rotational Structure and Excited-State Dynamics of Pyrene. J. Chem. Phys. 2009, 131, 224318.

(22) Hewett, K. B.; Shen, M.; Brummel, C. L.; Philips, L. A. High Resolution Infrared Spectroscopy of Pyrazine and Naphthalene in a Molecular Beam. J. Chem. Phys. 1994, 100, 4077-4086.

(23) Pirali, O.; Boudon, V.; Oomens, J.; Vervloet, M. Rotationally Resolved Infrared Spectroscopy of Adamantane. J. Chem. Phys. 2012, 136, 024310.

(24) Amirav, A.; Even, U.; Jortner, J. Energetics and Intramolecular Dynamics of the Isolated Ultracold Tetracene Molecule in Its First Excited Singlet State. J. Chem. Phys. 1981, 75, 3770-3793. 